\title{
Instrumentos políticos y financieros de la UE para la prevención de conflictos. El inacabado camino de la fragmentación hacia la integración ${ }^{1}$
}

\section{EU Political and Financial Instruments for Conflict Prevention. The Unfinished Process from Fragmentation to Integration}

\author{
Karlos Pérez de Armiño \\ Universidad del País Vasco (España)
}

Recibido: 22-10-20

Aceptado: 29-10-20

\section{Resumen}

Desde la década de 1990, la prevención de conflictos y la construcción de la paz se han convertido en objetivos relevantes de la acción exterior de la UE. Esta ha ido desarrollando un marco institucional compuesto por un entramado de organismos, políticas e instrumentos financieros, que le permite actuar en diferentes ámbitos y en todas las fases de un conflicto. Sin embargo, es un marco caracterizado por la fragmentación y la descoordinación. Por ello, la UE ha formulado los enfoques integral e integrado, y realizado varias reformas

\footnotetext{
${ }^{1}$ Trabajo realizado en el marco del proyecto RTI2018-098281-B-I00, convocatoria 2018 de "Proyectos de I+D Retos Investigación", subvencionado por el Ministerio de Ciencia e Innovación (MCI/AEI/FEDER, UE).

${ }^{2}$ (karlos.perezdearmino@ehu.eus). Profesor Titular de RRII, Universidad del País Vasco/ Euskal Herriko Unibertsitatea (UPV/EHU). Investigador Principal del Grupo de Investigación sobre Seguridad Humana, Desarrollo Humano Local y Cooperación Internacional (IT1037-16) del sistema universitario vasco (2016-2021). Algunas de sus publicaciones son: Diccionario de Acción Humanitaria y Cooperación al Desarrollo, Hegoa e Icaria, Barcelona, 2001. Disponible en: http:// www.dicc.hegoa.ehu.es/; Karlos Pérez de Armiño e Irantzu Mendia (eds), Seguridad humana. Aportes críticos al debate teórico y político, Tecnos, Madrid, 2013; "La tensión entre lo global y lo local en los procesos de construcción de la paz: aportes para una paz cosmopolita", en Caterina García Segura (dir.), La tensión cosmopolita. Avances y límites en la institucionalización del cosmopolitismo, Tecnos, Madrid, 2016, pp. 291-321 y Karlos Pérez de Armiño e Iker Zirion Landaluze (coords.), Pax Crítica. Aportes teóricos a las perspectivas de paz posliberal, Tecnos, Madrid, 2019.

ORCID: https://orcid.org/0000-0003-2422-700X.
} 
institucionales para dotar de mayor coherencia a sus políticas. Este objetivo se ha conseguido solo en parte, más en el ámbito técnico que en el político. Así, las políticas europeas están aún lejos de aprovechar todo su potencial, sobre todo en materia de prevención de las causas de los conflictos a largo plazo.

Palabras-clave: Unión Europea, prevención de conflictos, construcción de la paz, enfoque integrado, políticas.

\begin{abstract}
Since the 1990s, conflict prevention and peace-building have become relevant objectives of the EU's external action. Over time, it has developed an institutional framework made up of a network of agencies, policies and financial instruments, which allows it to act in different areas and in all phases of conflict. However, it is a framework characterized by fragmentation and lack of coordination. For this reason, the EU has formulated the comprehensive and integrated approaches, as well as implemented institutional reforms, to make its policies more coherent. This objective has only been partially achieved, more technically than politically. Thus, European policies are still far from taking advantage of their full potential, especially in terms of preventing the causes of conflict in the long term.
\end{abstract}

Key-words: European Union, Conflict Prevention, Peace Building, Integrated Approach, Policies.

\title{
1. Introducción
}

Desde la década de 1990, la prevención de conflictos y la construcción de la paz se han convertido en una parte integral y relevante de la acción exterior de la UE. Esta se ve a sí misma como una "constructora global de paz" (Castañeda, 2014:94) y, en general, como una potencia normativa que con sus políticas pretende difundir en el mundo determinados valores y principios, entre los que figuran la construcción de la paz y la prevención de conflictos, así como la democracia pluripartidista, los derechos humanos, el desarrollo sostenible y el multilateralismo (Manners, 2008). De este modo, su política exterior y de seguridad tradicionalmente ha tenido un carácter cosmopolita y transformador (Barbé y Morillas, 2019:758).

La UE ha conformado paulatinamente un marco normativo y político sobre prevención de conflictos y construcción de la paz, sustentado en diferentes documentos clave que han fijado principios y objetivos, e igualmente ha creado un denso entramado de instrumentos políticos, institucionales y financieros 
en la materia, que dependen de diferentes instituciones comunitarias. Aunque este marco ha cobrado cuerpo discursivo y político, hace solo una década era calificado aún como "naciente" y "embrionario" (Richmond et al, 2011:449).

Diferentes trabajos han subrayado que la UE tiene fundamentalmente tres ventajas comparativas que le proporcionan un potencial específico en este terreno, en comparación a otros actores internacionales: a) una variedad de instrumentos de actuación, tanto financieros como políticos; b) el peso internacional que le confiere ser el principal donante mundial de Ayuda Oficial al Desarrollo; y c) el hecho de no tratarse de un estado, lo que le facilita ser percibida como un actor más neutral en contextos de conflicto (Barrios, 2018:394).

En efecto, una de sus principales ventajas radica en que dispone de una amplia gama de instrumentos con los que actuar en todas las fases del ciclo del conflicto (antes, durante y después del mismo), tanto militares como, sobre todo, civiles. Entre ellos se incluyen el apoyo a las reformas políticas en países vecinos, la cooperación al desarrollo, las misiones de gestión de crisis, los acuerdos comerciales, etc.

Sin embargo, esa multiplicidad ha venido acompañada por problemas reiteradamente constatados, como son la fragmentación y la descoordinación institucional interna, y la falta de un liderazgo político claro en la materia. Consciente del problema, la UE ha adoptado a lo largo de las últimas décadas sucesivos enfoques conceptuales (como el enfoque integral y el enfoque integrado) y mecanismos operativos orientados a mejorar la coordinación. Estos esfuerzos parecen haber tenido un éxito limitado, pues han permitido avances hacia una mayor coherencia y articulación en el ámbito de la gestión operativa, pero no tanto en el ámbito de la dirección política.

El propósito de este artículo es analizar el complejo marco institucional de la UE en el campo de la prevención de conflictos y construcción de la paz, observando sus potencialidades y carencias así como su evolución histórica. A tal fin, en primer lugar esbozaremos algunas de sus características generales, incluyendo el potencial que representa su diversidad, la debilidad derivada de sus permanentes problemas de fragmentación, y las iniciativas adoptadas a lo largo del tiempo para superar estos. En segundo lugar, clasificaremos, describiremos y valoraremos los principales instrumentos políticos y financieros en este campo, que dependen de diferentes instituciones y órganos de la UE. En tercer lugar, analizaremos algunas otras carencias, tanto conceptuales como operativas, que aquejan a dicho marco, para finalizar con algunas conclusiones. 


\section{El marco europeo de prevención de conflictos y construcción de la paz. Hacia un enfoque integrado}

La UE carece de una institución o de un programa específicos dedicados a la prevención de conflictos y la construcción de la paz. En su lugar, dispone de un marco institucional constituido por una amplia diversidad de instrumentos políticos y financieros aplicados en ese campo, los cuales han ido conformándose y evolucionando a lo largo del tiempo. Son implementados por diferentes órganos de diversas instituciones de la UE, principalmente el Consejo, el Servicio Europeo de Acción Exterior (SEAE) y la Comisión.

Dicho entramado institucional se ha ido articulando en las dos últimas décadas mediante el impulso dado por diferentes documentos clave, que han ido conformando un marco normativo basado en la invocación de determinados principios y objetivos. Los dos primeros datan de 2001, y son la Comunicación de la Comisión Europea relativa a la prevención de conflictos (Comisión Europea, 2001), y el Programa de la UE para la prevención de conflictos violentos (Consejo Europeo, 2001). Este último, conocido como Programa de Gotemburgo, tuvo una importancia decisiva, pues fijó la construcción de la paz como una de las prioridades de la política exterior europea, si bien no logró precisar unas políticas específicas en la materia ni un reparto de responsabilidades entre las instituciones comunitarias (Duke, 2011).

Cabe destacar también la Estrategia europea de seguridad, adoptada por el Consejo en 2003 (Consejo de la UE, 2003). Además de ser la primera estrategia comunitaria en la materia, el documento fue clave para la formulación del carácter normativo y transformador de la política exterior de la UE (Barbé y Morillas, 2019:758), contemplando además la implicación de esta en diversas actividades de construcción de paz para afrontar las fuentes de inestabilidad. Más relevante aún ha sido el Tratado de Lisboa, en vigor desde 2009, pues afirma explícitamente que la acción exterior de la UE debe actuar de forma más coherente y aspirar a "preservar la paz, prevenir conflictos y reforzar la seguridad internacional" (Consejo Europeo, 2007, art. 21(2)). De este modo, estableció un mandato fuerte para que la UE y sus países miembros se impliquen en actividades de prevención de conflictos y de construcción de la paz (Juncos y Blockmans, 2018:131).

Un nuevo paso decisivo se dio con la adopción en 2013 por el Consejo Europeo de El enfoque integral para la acción externa en contextos frágiles $y$ afectados por el conflicto (Comisión Europea y Alta Representante, 2013). Como veremos, dicho enfoque integral (en la versión en inglés, comprehensive) buscaba superar la fragmentación institucional y mejorar la coordinación de todas las políticas en la materia. 
Tres años más tarde, en 2016, fue adoptada la Estrategia Global para la Política Exterior y de Seguridad de la Unión Europea (Unión Europea, 2016), que sustituyó a la antes citada Estrategia de Seguridad Europea de 2003. La Estrategia Global afirma su compromiso con la resolución de crisis y conflictos e introduce un nuevo concepto, el de enfoque integrado, entendido como una perspectiva multidimensional que pretende utilizar todas las políticas e instrumentos de los que dispone la UE. No obstante, como dicen Esther Barbé y Paul Morillas (2019:759-62), esta Estrategia Global ha supuesto una merma del carácter normativo, transformador y cosmopolita que tenía la de 2003, pues en su enfoque de política exterior han ganado peso las cuestiones de seguridad y defensa de la UE, así como los intereses de esta.

En base al marco normativo conformado por documentos como los arriba citados, la UE ha establecido una amplia gama de mecanismos institucionales, políticos y financieros que actúan en la materia. Algunos de ellos tienen una orientación esencialmente reactiva, pues se centran en la gestión a corto plazo de crisis o conflictos violentos (mediación, establecimiento del alto el fuego, misiones de mantenimiento de la paz, etc.). Por su parte, otros tienen objetivos de medio y largo plazo, pues se orientan bien a la prevención del conflicto armado minimizando sus causas, bien a la reconstrucción tras el fin del mismo, apoyando diferentes iniciativas (reformas políticas, desarrollo socioeconómico, protección de derechos humanos, justicia transicional, etc.). Aunque la UE dispone de instrumentos militares, como las misiones de la Política Común de Seguridad y Defensa (PCSD), su principal fortaleza reside en las políticas y actividades civiles (Richmond et al, 2011:452).

Una de las principales herramientas empleadas por la UE para la construcción de la paz en países vecinos es el estímulo que les supone la perspectiva de la integración o asociación con aquella, dando lugar a procesos en los que los países candidatos tienen que satisfacer progresivamente diferentes condiciones y reformas (Diez et al, 2006). De hecho, Richmond et al. consideran que la principal innovación del marco europeo de construcción de la paz consiste en la inclusión del concepto de integración regional, como acicate para los procesos de pacificación. Así pues, la actuación de la UE en materia de construcción de paz tiene más potencial e incidencia en el contexto del vecindario europeo, en el que puede utilizar tal perspectiva de la integración o asociación. En otros escenarios del mundo (Próximo Oriente, Sahel, Cuerno de África, etc.), su incidencia ha sido menos significativa y ha descansado esencialmente en aportes de ayuda material (Richmond et al, 2011:452, 257). 


\subsection{La diversidad de instrumentos: el esfuerzo por su integración}

Como decíamos, una de las principales potencialidades de la UE en el campo de la construcción de la paz radica en la diversidad de instrumentos de los que dispone, que le permiten actuar en todas las fases del conflicto armado y en multitud de ámbitos. Sin embargo, esa diversidad es también fuente de una fragmentación institucional agravada por una falta de coordinación y de coherencia en las políticas implementadas. Estos problemas han sido reiteradamente señalados no solo por trabajos académicos sino por documentos oficiales de la propia UE, la cual a lo largo del tiempo ha ido dando diferentes pasos para intentar paliarlos, especialmente la adopción en 2013 de un enfoque integral (comprehensive) en relación a la prevención de conflictos y gestión de crisis, y, en 2016, de un enfoque integrado, de carácter más ambicioso, en sustitución del anterior (Faleg, 2018).

En realidad, estos dos enfoques son resultado de un proceso que arranca ya en los años 90. Las primeras formulaciones en esta línea se basaron en el nexo seguridad-desarrollo, una idea en boga al acabar la Guerra Fría según la cual estas dos dimensiones se necesitan mutuamente y las actuaciones en ambas deben complementarse. A partir de este planteamiento básico, la UE y otros muchos actores internacionales fueron formulando enfoques que pretendían abarcar al conjunto de sus instituciones y acción de gobierno (whole-ofgovernment approaches). A ello contribuyó el estallido de crisis complejas en estados frágiles, con causas múltiples e impactos multidimensionales, lo que llevó a concluir que exigían una respuesta que combinara diferentes instrumentos (militares, diplomáticos y cooperación al desarrollo), con la cooperación de una amplia gama de actores a diferentes niveles (local, nacional y regional) a lo largo de todas las fases del conflicto (Debuysere y Blockmans, 2019:6).

Aunque el primer documento que propuso un enfoque comprehensivo de este tipo data de 1996, fue la Estrategia Europea de Seguridad de 2003 la que dio pleno peso político a la idea de que era precisa una mayor coordinación y coherencia entre los organismos y las políticas de la UE a fin de responder a los problemas de seguridad y de desarrollo, que están profundamente interrelacionados (Consejo de la UE, 2003).

Un hito decisivo en este proceso fue una comunicación conjunta de la Comisión y la Alta Representante de la UE para Asuntos Exteriores y Política de Seguridad, de 2013, titulada El enfoque integral para la acción externa en contextos frágiles y afectados por el conflicto (Comisión Europea y Alta Representante, 2013). El hecho de que fuera una comunicación conjunta de ambas instituciones testimonia la importancia dada al objetivo de que un enfoque integral (comprehensive) orientara toda la acción exterior de la 
UE, sobre todo en materia de prevención de conflictos y gestión de crisis. El documento entiende que tal enfoque integral se refiere, por un lado, a la adecuada coordinación de todas las instituciones, políticas e instrumentos de la UE, y, por otro, a una responsabilidad compartida de las instituciones de la UE y de sus Estados miembros.

Dicho documento buscaba superar la fragmentación institucional y la falta de coherencia en la acción exterior de la UE mediante una mayor coordinación política y operativa, en la línea del espíritu de integración estructural presente en el Tratado de Lisboa. Para ello, precisó una serie de medidas orientadas a mejorar la coherencia y efectividad de la política exterior y de la actuación en situaciones de conflictos y crisis, como por ejemplo: el desarrollo de análisis compartidos de la situación y de visiones estratégicas comunes, los compromisos a largo plazo, y la apuesta por la prevención desde la idea de una conexión entre la seguridad y el desarrollo.

La comunicación supuso un paso decisivo hacia la instauración de un enfoque integral, al ofrecer una interpretación conjunta del mismo. Sin embargo, también ha sido criticada por sus varias deficiencias. Por ejemplo, no proporcionó a los diferentes órganos comunitarios mecanismos o pautas específicas para implementar las recomendaciones formuladas y determinar dónde, cuándo, cómo y con quién actuar. Además, debido a la cercanía de las revueltas árabes de 2011, en realidad el enfoque integral prestó menos atención a la prevención de conflictos que a la gestión de crisis y conflictos (Faria 2014: 8-9; Hauck y Sherriff, 2013).

A la vista de tales deficiencias, otro paso decisivo se dio en 2016 con la introducción de un nuevo concepto, el del enfoque integrado. Este fue formulado en la Estrategia Global para la Política Exterior y de Seguridad de la Unión Europea (Unión Europea, 2016), la cual muestra su compromiso con la resolución de crisis y conflictos, principalmente en las áreas vecinas del Sur y Este, pero sin renunciar a actuar en zonas más lejanas. Para ello formula el enfoque integrado a conflictos y crisis externas, que tiene cuatro características: a) multidimensional, por cuanto se deben utilizar todos los instrumentos y políticas de los que la UE dispone para la prevención y gestión de conflictos (diplomacia, misiones y operaciones de la PCSD, cooperación al desarrollo y ayuda humanitaria); b) multifase, pues implica una actuación en todas las fases del ciclo del conflicto, antes (prevención), durante (resolución y estabilización) y después del mismo (reconstrucción, evitando un abandono prematuro del escenario); c) multinivel, ya que la complejidad de los conflictos exige actuar a nivel local, nacional, regional y global; y d) multilateral, dado que la construcción de la paz de la UE debe basarse en una colaboración amplia y duradera con diferentes actores, desde otras organizaciones internacionales hasta la sociedad civil en el terreno. 
Además, el enfoque integrado identifica una serie de áreas prioritarias en las que actuar, como son la realización de análisis compartidos, la sensibilidad al conflicto de las diversas actuaciones, el apoyo a la mediación, la reforma del sector de seguridad, el sistema de alerta temprana de la UE, el enfoque preventivo de crisis y la acción temprana, la respuesta a las crisis (mediante la PCSD y la ayuda humanitaria), la estabilización y la justicia transicional (Debuysere y Blockmans, 2019:12).

El enfoque integrado encierra un alcance algo más ambicioso y holístico que el anterior enfoque integral: tiene una formulación más política, a más largo plazo y con un mayor esfuerzo operativo (Debuysere y Blockmans, 2019:13). Así, representa un mayor desafío institucional, pues si el enfoque integral proponía simplemente una coordinación horizontal, el integrado exige profundizar en la cooperación entre actores y romper con los compartimentos estancos (Faleg 2018:4). Más que añadir elementos nuevos al enfoque integral, su aporte radica en que afirma la necesidad de expandirlo mediante una mejora de su carácter multinivel, multilateral, multifase y multidimensional (Tardy 2017: 2). En otras palabras, este enfoque busca aprovechar uno de los principales valores añadidos de la UE: que el hecho de disponer de una variada gama de capacidades e instrumentos tanto militares como civiles, le permite dar una respuesta multidimensional a los conflictos, garantizando una secuencia continua de medidas que cubra todas las fases de una crisis (Churruca, 2017:959).

Además, es reseñable que el enfoque integrado representa un avance conceptual, por cuanto enfatiza la importancia de la prevención de las causas de los conflictos, incorpora algunas perspectivas de seguridad no tradicionales (como enfoques de abajo arriba y otros propios de las perspectivas no liberales de construcción de la paz), y se formula como un medio para promover la seguridad humana (Debuysere y Blockmans, 2019: 6, 11).

El enfoque integrado ha sido experimentado, por ejemplo, en los Balcanes occidentales, una región que ha sido crucial en el desarrollo de las políticas de paz de la UE (Juncos y Blockmans, 2018:132). Es importante observar que, para hacerlo operativo, la UE ha acometido varias reformas institucionales, como la creación en 2017 de la división PRISM y en 2019 de la Dirección ISP. Estas y otras medidas, que veremos, sin duda han contribuido a mejorar la coordinación entre las instituciones comunitarias. Sin embargo, todavía perduran las dificultades para superar la fragmentación y el funcionamiento autónomo de diferentes instancias y políticas de la UE. 


\section{Instrumentos para la prevención de conflictos y la construccion de la paz}

Los numerosos instrumentos políticos, institucionales y financieros utilizados por la UE en este campo dependen de varias de sus instituciones, principalmente el Consejo, el Servicio Europeo de Acción Exterior (SEAE) y la Comisión. Veamos los más importantes.

\subsection{Instrumentos del Consejo de la UE}

La configuración del Consejo más concernida por los temas de construcción de paz y prevención de conflictos es el Consejo de Asuntos Exteriores, por cuanto le competen la política exterior (PESC), la defensa y seguridad (PCSD), el comercio, la cooperación para el desarrollo y la ayuda humanitaria.

En efecto, es el principal responsable de la Política Exterior y de Seguridad Común (PESC) de la UE, la cual incluye múltiples actividades relativas a la construcción de la paz, tales como: el diálogo político con terceros países, la participación en conversaciones de paz, las declaraciones conjuntas sobre problemas internacionales, las sanciones políticas o económicas, etc. El Consejo es el principal responsable también de la Política Común de Seguridad y Defensa (PCSD), en la que se enmarcan las políticas y actuaciones con implicaciones militares y de defensa, como por ejemplo la treintena de misiones y operaciones civiles y militares realizadas con diversos objetivos (estabilización militar; desarme, desmovilización y reintegración de excombatientes; reforma del sector de seguridad; etc.).

Para cumplir estas funciones, el Consejo de la UE cuenta con diferentes órganos. Destaca el Comité Político y de Seguridad (CPS), compuesto por representantes de los veintiocho Estados miembros, y encargado de elaborar y supervisar las políticas de la PESC. Así, bajo la autoridad del Consejo y del Alto Representante, el CPS lleva a cabo el control político y la dirección estratégica de las misiones, en particular de la gestión civil de crisis. Con este concepto la UE se refiere a la gestión no militar de crisis en el marco de las misiones de la PCSD, mediante actividades relativas, por ejemplo, al refuerzo de la policía, del estado de derecho y de la administración civil en contextos frágiles; la reforma del sector de seguridad; y funciones de monitoreo

En suma, dadas las citadas funciones desempeñadas por el Consejo, son los Estados miembros los que marcan la dirección estratégica y definen los aspectos más relevantes de la respuesta de la UE a los conflictos, en particular los relativos a cuestiones duras de seguridad. Esto conlleva que tal respuesta viene condicionada, y a veces obstaculizada, por la dificultad de consensuar posturas comunes entre los Estados, habida cuenta de sus diferentes intereses de política exterior y posicionamientos sobre la construcción de la paz. 


\subsection{Instrumentos del Servicio Europeo de Acción Exterior (SEAE)}

Tras su creación por el Tratado de Lisboa como servicio diplomático de la UE, el SEAE juega un papel destacado en la respuesta europea a los conflictos. En efecto, el SEAE tiene como cometido asegurar la coherencia de la acción exterior de la UE mediante diferentes funciones: programación y gestión de la misma, coordinación de los Estados miembros en terceros países y representación diplomática de la UE en el exterior.

A tal fin, el SEAE asiste al Consejo con la preparación de sus decisiones, por ejemplo el despliegue de las misiones PCSD. Además, contribuye a la programación y gestión de diferentes instrumentos aplicables a la construcción de la paz, en colaboración por ejemplo con la Dirección General de Cooperación Internacional y Desarrollo (DG DEVCO), al tiempo que participa en la elaboración de la asignación financiera a terceros países y de los documentos estrategia país, que delimitan las relaciones con aquellos.

Además de dichas funciones, el SEAE tiene la responsabilidad específica de diferentes instrumentos, como los siguientes.

\subsubsection{Instrumento en pro de la Estabilidad y la Paz (IEP/ISP).}

Se trata del principal instrumento del SEAE de asistencia financiera y técnica para la construcción de la paz y la prevención de conflictos. Surgió en 2015 para sustituir al Instrumento en pro de la Estabilidad, que había sido creado para facilitar una respuesta rápida a las crisis. Tal cambio de nombre reflejó una apuesta por dar mayor relevancia a la construcción de la paz como ámbito específico (Barrios, 2018:397). En el período 2015-2020 ha contado con un presupuesto de 2340 millones de euros, con el objetivo de responder a crisis emergentes, y contribuir a la estabilidad, la prevención de conflictos y la reconstrucción posconflicto. Para ello financia una amplia gama de actividades en diversos campos: derechos humanos, democracia, desarrollo, participación de la sociedad civil, igualdad de género, justicia transicional, reincorporación de excombatientes, etc.

Su financiación se orienta a tres apartados principales. El primero, que absorbe la mayoría del presupuesto (70\%), se destina a la prevención de crisis y a la respuesta a crisis emergentes o fuerte desestabilización. El segundo (20\% del presupuesto) se centra en la asistencia para afrontar amenazas globales y transrregionales a largo plazo (amenazas a infraestructuras críticas, terrorismo, crimen organizado, etc.). Un tercer apartado se dedica a la "Asistencia para prevención de conflictos, construcción de paz y preparación de crisis". Aunque es el más limitado ( $9 \%$ del presupuesto), tiene la singularidad de que se refiere expresamente a esos dos ámbitos, en los que financia actividades relativas, por 
ejemplo, a la alerta temprana y el análisis de riesgos y conflictos; la construcción de confianza, mediación, diálogo y reconciliación; el despliegue de misiones civiles de estabilización; y la reconstrucción posconflicto (EPLO, 2014:2-6).

Como podemos apreciar, la mayor parte de este instrumento se dedica a la respuesta a crisis que han estallado o están agravándose; pero, sin embargo, merece destacar que una parte se destina a la prevención de conflictos, la construcción de la paz y la preparación ante las crisis. Es también reseñable que el reglamento de este instrumento incluye una referencia explícita a la consulta con la sociedad civil de los países concernidos.

\subsubsection{Direcciones ligadas a la PESC y la PCSD}

En el marco del SEAE existen además varias direcciones ligadas a la PESC y la PCSD. Destacan las siguientes:

\section{a) Dirección del Enfoque Integrado para la Seguridad y la Paz (ISP).}

Ha sido creada recientemente, en marzo 2019, como órgano clave de la UE para aplicar el enfoque integrado en la materia. Su nacimiento ha sido el colofón de un proceso habido durante años para mejorar la coordinación entre las instituciones comunitarias en este campo, que se ha traducido en la creación y posterior desaparición de diferentes órganos.

En efecto, con su creación se han desmantelado y fusionado otros dos órganos centrados respectivamente en funciones de planificación estratégica y de implementación técnica: la Dirección de Gestión de Crisis y Planificación (CMPD), núcleo encargado de la planificación político-estratégica, civil y militar, de las misiones de la PCSD, esto es, de las operaciones de mantenimiento de la paz y humanitarias de la UE; y la División para la Prevención de Conflictos, Estado de Derecho/SSR, Enfoque Integrado, Estabilización y Mediación (PRISM). La división PRISM (que había sido creada en 2017 en sustitución de otro órgano, la SECPOL.2) se constituyó en el referente técnico y punto focal de la respuesta de la UE en las distintas fases del ciclo del conflicto, pero adolecía de un escaso peso institucional en el organigrama del SEAE, lo que hizo ver la necesidad de crear la Dirección ISP (Debuysere y Blockmans, 2019: 21-3).

El hecho de haber constituido la ISP con el rango de dirección ha sido un paso importante, pues le ha dotado de un mayor peso para la interlocución con otros órganos del mismo nivel, así como también de una mayor capacidad de coordinación y gestión operativa, y de una cadena de mando más clara. De esta forma, en colaboración con otros servicios de la SEAE e instituciones de la UE, su cometido es coordinar las actuaciones de la UE en materia de seguridad, 
desarrollo y diplomacia, orientadas a unos objetivos comunes en el marco del enfoque integrado, y en todas fases del conflicto. Así, por ejemplo, alberga las reuniones de gestión de crisis con la implicación de todas las divisiones del SEAE y direcciones generales de la Comisión concernidas (ECHO, DEVCO, NEAR).

La Dirección ISP está estructurada en cinco divisiones. La ISP.1 se centra en procesos de desarme, desmovilización y reintegración (DDR), y en la reforma del sector de seguridad, la seguridad humana y la generación de lecciones aprendidas. La ISP. 2 gestiona el sistema de alerta temprana y proporciona análisis sobre conflictos, así como apoyo en materia de diálogo y mediación. La ISP.3 está encargada de la planificación de la respuesta de la UE a situaciones de crisis, en particular de las misiones civiles y militares de la PCSD. La ISP.4 se ocupa de la protección de ciudadanos europeos en crisis en el exterior (EPLO 2020:17). Por último, la ISP.5 ha sido creada en mayo de 2020 y se encargará de la gestión general del Fondo Europeo de Paz (EPF), un fondo extrapresupuestario que entre 2021 y 2027 financiará las acciones de la PCSD que tengan implicaciones militares o de defensa. Esto incluirá el apoyo a ejércitos de otros países, con capacitación y la provisión de armamento letal, lo cual, como señala Hauck (2020), ha suscitado críticas.

Como podemos apreciar, la Dirección ISP ha asumido la gestión del sistema de alerta temprana que la UE ha ido desarrollando en materia de conflictos. Tal sistema recopila información de diferentes fuentes, incluida la sociedad civil local, para identificar el riesgo de conflicto. Aunque es un mecanismo que genera análisis útiles, numerosos estudios constatan que existen dificultades para traducirlos luego en acciones de respuesta temprana (Davis, 2018:163, 165).

En suma, la ISP cubre un amplio abanico de funciones y ha representado un avance de cara a aplicar el enfoque integrado. Sin embargo, adolece de diferentes limitaciones que dificultan que pueda implementarlo de forma efectiva. En primer lugar, el hecho de que el nombre de la división ISP ponga el concepto seguridad por delante del de paz parece expresar las prioridades del enfoque integrado: de hecho, buena parte de su personal se dedica a la planificación estratégica de la PCSD y la estabilización, y una parte mucho menor a la prevención y la mediación en conflictos. En segundo lugar, aunque es cierto que la ISP cubre tanto la dimensión política, con la planificación de políticas en la materia, como la dimensión operativa, con las misiones civiles y militares de la PCSD, lo cierto es que su capacidad de integración se constata en el ámbito burocrático pero menos en el político: esto es, ha permitido fusionar las funciones operativas, pero no el nivel operativo con el político. De hecho, las actividades de la ISP, como son las reuniones que organiza en situaciones de crisis, apenas cuentan con la participación de los Estados miembros, por lo 
que no sirven para integrar sus visiones ni generar posturas a las que se sientan vinculados. A esto se añade que la ISP no ha permitido integrar las estructuras de la PCSD y otras de respuesta a las crisis con las direcciones geográficas de la SEAE. En suma, la Dirección ISP no puede verse como una varita mágica para poder aplicar un enfoque del "conjunto de Europa" a los conflictos (Debuysere y Blockmans, 2019:21, 23).

b) Dirección para Asuntos de Derechos Humanos, Mundiales y Multilaterales (GLOBAL)

Esta Dirección ejerce múltiples funciones pertinentes a un enfoque preventivo de los conflictos y está articulada en seis divisiones, encargadas de diferentes ámbitos como: derechos humanos, democracia y observación electoral, migración y seguridad humana, cooperación al desarrollo y relaciones multilaterales con la ONU y otras organizaciones internacionales.

Cabe destacar que, entre otras múltiples tareas, esta Dirección ejerce varias relativas a la perspectiva de género en el ámbito de la construcción de la paz. Así, la División de Derechos Humanos (GLOBAL 1) se ocupa de la integración de la perspectiva de género en las políticas de derechos humanos, así como de los temas relativos a la Resolución 1325 del Consejo de Seguridad sobre Mujeres, Paz y Seguridad. No obstante, según EPLO (2020:17-18), la escasez de personal dedicado a este ámbito dificulta que la SEAE pueda integrar adecuadamente en sus políticas la equidad de género. Además, algunas autoras han señalado que la implementación de la agenda de género en la construcción de la paz de la UE ha sido parcial y dispersa, y que se basa en una visión de género débil y conservadora, pues se limita a incluir a las mujeres en las estructuras pero sin cuestionar estas ni las relaciones de poder (Martín de Almagro, 2017:2).

c) Otras estructuras e instrumentos del SEAE

Además de los citados, el SEAE cuenta con otros órganos e instrumentos operativos en el campo de la prevención de conflictos, por ejemplo:

-La Dirección de Política de Seguridad y Defensa (SECDEFPOL). Tal y como indica su nombre, se ocupa de cuestiones "duras" de seguridad, en parte relacionadas con la prevención de conflictos y la construcción de la paz.

-Las direcciones regionales. Al estar encargadas de la formulación de las políticas de la UE tanto para diferentes regiones como para sus países, cumplen un papel relevante en el caso de zonas golpeadas por conflictos. 
-La Capacidad Civil de Planificación y Ejecución (CPCC). Es responsable de la planificación, implementación y revisión de la "gestión civil de crisis", esto es, actividades no militares en misiones de la PCSD.

-La Capacidad Militar de Planificación y Ejecución (MPCC). Es responsable de la planificación operativa e implementación de las misiones militares de la UE denominadas "no ejecutivas" (no de combate); en concreto, de las misiones de formación implementadas en Somalia, República Centroafricana y Mali.

-La División de Politica y Planificación Estratégica, responsable de la planificación estratégica a largo plazo del SEAE.

-Las Delegaciones de la UE. Se trata de las 141 delegaciones y oficinas de la UE por todo el mundo. Reciben instrucciones tanto del Alto Representante como de la Comisión, y cuentan con personal tanto del SEAE como de aquella, en concreto personal de DEVCO encargado de los proyectos de cooperación. Esto da lugar a que a veces coexistan culturas institucionales diferentes, causando una merma en la eficacia (Barrios, 2018:392). Las delegaciones cumplen funciones importantes en el campo de la prevención de conflictos, como son la realización de análisis sobre el contexto de conflicto, el respaldo a procesos de diálogo político y el apoyo a proyectos de la sociedad civil. No obstante, según Davis (2018:165), el potencial de las delegaciones en materia de construcción de paz está infrautilizado.

-Los Representantes Especiales de la UE. Son nombrados por el Consejo de la UE a propuesta del Alto Representante, a quien reportan, y tienen por objeto incrementar la presencia activa de la UE en regiones o países conflictivos, con actividades de diplomacia itinerante, mediación y otras orientadas a la construcción de la paz, la estabilidad y el estado de derecho. A principios de 2020 estaban en activo ocho representantes. Suelen contar con un grupo de asesores que actúan en contacto con la sociedad civil.

-Los Fondos Fiduciarios de la UE. Se trata de mecanismos especiales de asignación de fondos que agrupan un alto volumen de recursos aportados por diferentes donantes a fin de proporcionar una respuesta común, rápida y flexible en contextos de crisis o poscrisis. El SEAE participa en la decisión de su establecimiento. Su regulación data de 2013, habiéndose establecido cuatro fondos fiduciarios orientados a la República Centroafricana, Siria, África (para el afrontamiento de causas de la migración irregular) y Colombia. El fondo Fiduciario de Africa, en 
particular, ha sido criticado por su falta de transparencia en la toma de decisiones y en los criterios para que las organizaciones de la sociedad civil puedan solicitar tales fondos (EPLO, 2020:25).

-El Asesor Principal sobre Género y sobre la Implementación de la Resolución 1325 del Secretario General de las NNUU sobre Mujeres, Paz y Seguridad. Creado en 2015, reporta al Secretario General del SEAE y tiene como función coordinar las diferentes instituciones de la UE, así como a esta con otros actores internacionales, en relación a las cuestiones de género y a dicha resolución, para que ganen peso en la acción exterior de la UE.

Además, cabe añadir que el SEAE incide también en la prevención de conflictos y la construcción de la paz a través de dos tipos de prácticas:

-Las cláusulas de condicionalidad incluidas en Acuerdos de Asociación (AdA), o tratados bilaterales sobre comercio e inversiones firmados con otros países. Estos tratados suelen incluir componentes en materia de cooperación y diálogo político, con cláusulas en defensa de los derechos humanos y la promoción de la democracia. No obstante, diferentes voces critican que la cooperación y el diálogo político suelen ser secundarios respecto al componente comercial e inversor; y que los acuerdos con frecuencia tienen un impacto lesivo sobre las condiciones socioeconómicas de las poblaciones locales, contradiciendo el objetivo de la construcción de la paz.

-Las Iniciativas de diálogo político, mediación y diplomacia preventiva. El SEAE implementa una amplia gama de medidas que van desde el incentivo y la persuasión a terceros actores, hasta la crítica pública y las sanciones. Un ámbito que ha experimentado un notable desarrollo en la última década es el de la mediación, gracias a la formulación de una política en la materia plasmada en un documento específico (Council of the EU, 2009). Además, en la nueva Dirección ISP se ha establecido una División sobre Prevención de Conflictos y Apoyo a la Mediación (ISP.2) que proporciona apoyo especializado en materia de diálogo y mediación a las delegaciones y las divisiones geográficas de la UE. No obstante, el SEAE lleva a cabo escasa diplomacia preventiva, en parte por la dificultad de llegar a posiciones comunes entre los Estados miembros (Davis, 2018:165). 


\subsection{Instrumentos de la Comisión Europea}

Como es sabido, la Comisión es la encargada de ejecutar las políticas de la UE en temas comunitarizados, programar su trabajo y gestionar su presupuesto. Cuenta con 5 direcciones generales que actúan en el ámbito de las relaciones exteriores, así como con diferentes direcciones e instrumentos operativos en la prevención de conflictos y la construcción de la paz.

\subsubsection{Servicio de Instrumentos de Política Exterior (FPI)}

El FPI, creado a la vez que la SEAE, es la única estructura de la Comisión que reporta directamente al Alto Representante, en su papel de Vicepresidente de la Comisión. En su seno cabe destacar la FPI.2 o Unidad para el Instrumento en pro de la estabilidad y la paz (IcSP), responsable de la planificación y administración financiera de dicho instrumento, el cual sufraga una amplia gama de actividades para la respuesta a crisis emergentes, el afrontamiento de amenazas globales, la prevención de conflictos y la reconstrucción posconflicto. Esta tarea se realiza en coordinación con el SEAE, encargado de su planificación estratégica multianual, con la Dirección General para Cooperación Internacional y Desarrollo (DG DEVCO), encargada de la ejecución de algunos de los programas, y con otros servicios de la Comisión. Por su parte, otra unidad, la FPI.3, está encargada de la gestión financiera de las misiones civiles PCSD.

Cabe señalar que la creación del FPI no se contempló en el Tratado de Lisboa, sino que surgió como "resultado de una lucha institucional entre el EEAS y la Comisión sobre quién gestionaría los instrumentos financieros relativos a la PESC". Aunque ha mejorado el nivel de cooperación entre el FPI y el SEAE, perdura el problema de que los recursos humanos capacitados en materia de conflictos están separados entre el SEAE y la Comisión (EPLO, 2020:22).

\subsubsection{Dirección General para Cooperación Internacional y Desarrollo (DG DEVCO)}

La DG DEVCO es la encargada de la formulación e implementación de las políticas, programas y proyectos de desarrollo de la UE, para lo cual parte de su personal está en sus delegaciones en el exterior. La UE, como otros actores internacionales, ha asumido la idea de que existe un estrecho vínculo entre el desarrollo y la paz, y que por tanto la cooperación al desarrollo es un instrumento útil para la construcción de la paz. Así se constata en numerosos documentos, como la Estrategia de Seguridad Europea de 2003. 
Sin embargo, diferentes voces cuestionan que la UE esté llevando esta perspectiva a la práctica. Tradicionalmente viene prestando más atención y recursos a la respuesta de crisis a corto plazo que a la prevención de los conflictos a largo plazo mediante políticas de desarrollo y reformas políticas estructurales. De hecho, la mayoría de sus actuaciones en contextos de conflicto no abordan las causas políticas subyacentes, sino que adoptan un enfoque técnico y operativo para capacitar el Estado (Bell, 2009:11, 16) y garantizar la estabilidad en base a acuerdos con las élites nacionales. También se ha señalado que las políticas de la UE asumen un paradigma liberal del desarrollo, basado en el crecimiento y la integración comercial, que en ocasiones puede aliviar los conflictos pero, en otras, puede incrementar las desigualdades y las tensiones (Pogodda et al, 2014:239).

Por otro lado, según la organización EPLO, diferentes políticos europeos del Parlamento y la Comisión implicados en el campo del desarrollo no son conscientes de las nuevas perspectivas sobre tal vinculación de la cooperación al desarrollo con la construcción de la paz, o son hostiles a la misma. A su vez, otros políticos de esas instituciones y de los Estados miembros pretenden utilizar el presupuesto de la cooperación al desarrollo para proporcionar formación y equipamiento a fuerzas de seguridad y ejércitos de países socios, lo cual sería problemático (EPLO, 2020:25).

DEVCO abarca varias unidades que son relevantes en el ámbito de la construcción de la paz. Destaca la Unidad Resiliencia y Fragilidad (DEVCO.B.2), encargada de transversalizar la sensibilidad al conflicto, la construcción de la paz y la resiliencia en las políticas de cooperación europeas en escenarios frágiles. A tal fin, apoya y asesora a diferentes unidades geográficas y unidades temáticas de DEVCO centradas en diversos campos (igualdad de género, derechos humanos, gobernanza democrática, etc.).

Además, DEVCO participa en la programación y es la responsable de la implementación de varios instrumentos financieros relevantes en este ámbito, como son los siguientes.

\section{a) El Instrumento de Cooperación al Desarrollo (ICD)}

El ICD ha contado con un presupuesto de 19.700 millones de euros para el período 2014-2020. Su Reglamento señala que uno de los temas que debe ser transversalizado en las políticas de desarrollo de la UE es la paz. Igualmente, señala que la prevención de conflictos es una de las áreas de cooperación que deben apoyarse mediante los programas geográficos, para lo cual estos deben abordar las causas raíces de los conflictos, promover el diálogo, la participación y la reconciliación, así como apoyar la cooperación y reforma política en materia de seguridad y justicia (EPLO, 2014:6). 


\section{b) Fondo Europeo de Desarrollo (FED)}

Con una dotación de 30.500 millones de euros para 2014-2020, el FED puede financiar actividades de prevención de conflictos a corto y a largo plazo, en diferentes lugares del mundo, que hayan sido incluidas en los documentos de estrategia país.

\section{c) Iniciativa Europea para la Democracia y los Derechos Humanos (IEDDH)}

Este instrumento financiero, creado en 2005, ha contado con un presupuesto de 1.333 millones de euros entre 2014 y 2020. DEVCO lidera su ejecución, consultando al SEAE. Sirve para financiar diferentes actividades, muchas de ellas en colaboración con organizaciones de la sociedad civil, en ámbitos como: la democratización, los derechos humanos, la reconciliación social, y las misiones de observación electoral para garantizar unas elecciones pacíficas y transparentes.

Como hemos visto, los derechos humanos constituyen un objetivo esencial en los documentos programáticos de la política exterior europea y de la prevención de conflictos. Se asume que su promoción, así como las medidas de democratización, ayudarán a aliviar los agravios políticos de diferentes grupos sociales y étnicos, y por tanto a construir la paz. Ahora bien, diferentes fuentes advierten de la brecha existente entre el discurso y la práctica de la UE en esta materia: en las actuaciones de construcción de la paz y de seguridad, los derechos humanos reciben insuficientes recursos humanos, materiales e institucionales, menos que los dedicados a campos como la lucha antiterrorista o la reforma del sector de seguridad (Hadden, 2009).

\subsubsection{Dirección General de Política de Vecindad y Negociaciones de Ampliación (DG NEAR)}

Creada en 2015, se encarga de gestionar la asistencia técnica y financiera de la UE a los países vecinos y a aquellos susceptibles de integración en la misma. Muchos de los países vecinos de Europa, en su flanco Este y Sur, sufren prolongados conflictos armados, los han sufrido o tienen un alto riesgo de estallido de la violencia, en tanto que varios países candidatos de los Balcanes Occidentales están en proceso de reconstrucción de posguerra. Así pues, muchas de las políticas de la DG NEAR, si se implementan adecuadamente, tienen el potencial de contribuir a la prevención de conflictos y la construcción de la paz. Tales políticas se materializan mediante dos instrumentos financieros. 
a) El Instrumento Europeo de Vecindad (IEV), que ha contado con un presupuesto de 15.433 millones de euros entre 2014 y 2020. Su objetivo es establecer una relación especial con los países vecinos al Este y Sur de la UE, basada en los valores de la democracia, el estado de derecho y los derechos humanos. Para ello, incluye medidas de apoyo a las reformas democratizadoras, así como otras para promover la construcción de confianza y buena vecindad, la seguridad y la solución de conflictos, y la reconstrucción posbélica.

b) El Instrumento de Ayuda de Preadhesión (IPAII), dotado con 11.700 millones de euros entre 2014 y 2020. Sirve para gestionar la relación bilateral con los países de los Balcanes Occidentales que son candidatos a la integración, o pueden serlo, así como para apoyar las reformas que tienen que realizar en materia de estado de derecho, gobernanza económica y reforma de la administración pública.

La DG NEAR lleva a cabo un seguimiento del progreso de las relaciones con los países vecinos y candidatos, así como de las reformas por ellos implementadas, en base a los criterios fijados en sus acuerdos con la UE. Tal seguimiento se hace mediante informes, en los cuales suelen incluirse aportes de la sociedad civil de tales países.

\subsubsection{Dirección General de Comercio (DG Trade)}

Esta Dirección General está encargada de la Política Comercial Común (PCC) de la UE con terceros países, lo cual abarca el comercio externo de bienes y servicios, y las inversiones directas. Está encargada de preparar y negociar los acuerdos comerciales y de inversión con terceros países, tales como los Acuerdos de Asociación Económica (AAE) con países en vías de desarrollo, en los cuales, para paliar su posible impacto negativo, se incluyen clausulas sobre los derechos humanos, los conflictos y la protección medioambiental.

El comercio es un ámbito de crucial importancia para los conflictos: puede contribuir a prevenirlos al promover relaciones pacíficas entre países o el crecimiento de la economía; pero también puede alimentar sus causas estructurales si consolida modelos económicos basados en grandes desigualdades o en el despojo de recursos locales. Por otro lado, hay que recordar que el Tratado de Lisboa, artículo 207(1), integró la PCC como parte de la acción exterior de la UE y estipuló que debe implementarse conforme a sus principios y objetivos, entre los cuales figura la promoción de la paz en el mundo.

Sin embargo, como señala EPLO (2020:32), su potencial contribución viene limitada por varias circunstancias. La mayoría de los funcionarios de esta Dirección carecen de la experiencia para analizar los impactos no económicos de la política comercial y con frecuencia son reacios a que esta sirva a otros 
objetivos de la política exterior de la UE, una postura respaldada por muchos Estados miembros. Además, esta DG es más poderosa que las otras centradas en la acción exterior y la prevención de conflictos, pues, no en vano, los Estados miembros le han confiado la defensa de sus intereses en la negociación de los acuerdos. Por ello, en las negociaciones internas de la Comisión tiene mayor peso que otras y resulta difícil influir en ella para que incorpore el objetivo de la construcción de paz en sus políticas. De hecho, no considera que el comercio forme parte del enfoque integrado de la UE en relación a los conflictos y, además, rechaza el papel del SEAE en la coordinación del conjunto de la acción exterior de la UE.

\subsubsection{Dirección General de Protección Civil y Operaciones de Ayuda Humanitaria Europeas (ECHO)}

La mayor parte de sus proyectos de ayuda humanitaria se implementan en países en conflicto armado o que han salido recientemente de él. Así, ECHO ha asumido la importancia del llamado triple nexo, o nexo acción humanitariadesarrollo-paz, según el cual las políticas de acción humanitaria, cooperación al desarrollo y construcción de una paz sostenible tienen que reforzarse entre sí. Esta perspectiva, con amplio respaldo en la academia y los actores internacionales, puede contribuir a integrar diferentes políticas al servicio del afrontamiento de las causas de los conflictos. Sin embargo, algunos sectores temen que acabe erosionando los principios humanitarios (independencia, neutralidad e imparcialidad) y sometiendo la acción humanitaria a la agenda de seguridad (EPLO, 2020:35).

\subsection{Funciones del Parlamento Europeo}

El Parlamento tiene poderes bastante limitados en política exterior, a lo que se añade que algunos parlamentarios son renuentes a que la UE juegue un papel en ella o, simplemente, no están interesados en la construcción de la paz (EPLO, 2020:37-9). Aún así, puede cumplir algunas funciones en tres ámbitos: el legislativo, pues tiene poder de codecisión en campos de competencia comunitaria, como las políticas de desarrollo y los acuerdos comerciales; el presupuestario, pues su poder de codecisión sobre todo el presupuesto le da cierta influencia sobre la política exterior; y el consultivo y de supervisión, que son las que puede ejercer en relación a la PESC y la PCSD, den las que las decisiones son tomadas básicamente por el Consejo. Esta labor de seguimiento se realiza mediante dos debates anuales sobre los avances de tales políticas, y la aprobación de sendos informes; así como mediante debates y audiencias públicas con los comisarios, el Alto Representante y funcionarios del SEAE, a 
los que se les formulan preguntas y recomendaciones (Debuysere y Blockmans, 2019:26).

Cabe añadir que el Parlamento organiza, junto al SEAE, las Misiones de Observación Electoral de la UE, en la que suelen participar parlamentarios, las cuales tienen un potencial infrautilizado, según Davis (2018:164). Además, el Parlamento ha ido asumiendo funciones de mediación política con parlamentos de otros países, que se han consolidado con la creación de una Unidad de Mediación y Diálogo (Debuysere y Blockmans, 2019:27).

\subsection{Instrumentos del Banco Europeo de Inversiones (BEI)}

Una parte de los créditos del BEI, banco público de la UE, se destinan a financiar proyectos en el exterior, como la construcción de grandes infraestructuras. En los últimos años el BEI ha adoptado enfoques de sensibilidad al conflicto, buscando que sus inversiones no agraven los conflictos y, cuando sea posible, contribuyan indirectamente a la construcción de la paz. Sin embargo, sus informes de actividad no incluyen menciones a la sensibilidad al conflicto. Además, los Estados suelen ser reticentes a frenar determinados proyectos cuando se ven afectadas empresas de su país (EPLO, 2020:48-9).

\section{Problemas de los instrumentos de construcción de la paz de la UE}

Como hemos visto, en las últimas décadas la UE ha ido conformando un marco de construcción de paz basado en una amplia gama de instrumentos políticos y financieros. Sin embargo, diferentes estudios señalan que dicho marco adolece de diferentes problemas que limitan su eficacia, entre los que destacan los siguientes.

\subsection{Confusión conceptual}

Existe en la UE una falta de claridad en la definición de los principales términos que utiliza en este campo, lo que afecta a su aplicación (EPLO 2020:15). Por un lado, la UE tiene cierta confusión sobre el término prevención de conflictos, pues unas veces la entiende como una serie de actividades específicas (alerta temprana, mediación, etc.) para evitar la violencia en un momento dado, pero otras veces la interpreta como una forma que tiene la UE de implicarse en el mundo mediante el apoyo a transformaciones de largo plazo, algo sumamente difuso y cuyo impacto es casi imposible constatar (Davis, 2018:157). 
Por su parte, el de construcción de la paz, más reciente y menos habitual en el vocabulario de la UE, se emplea de forma imprecisa, pues no hay ningún documento de la Comisión o del Consejo que lo defina. Esto lleva a que diferentes instituciones europeas la interpreten de formas diversas y, a veces, contradictorias (Barrios, 2018:389). Fruto de esta indefinición, con frecuencia se mezcla con otros conceptos, como el de estabilización, cuando ambos pueden resultar contrapuestos: si la estabilización supone aplacar la violencia física mediante el apoyo a estructuras o regímenes opresivos, el resultado puede ser un aplazamiento o agravamiento del conflicto.

\subsection{Carencia de una estrategia clara, compartida y coherente de construcción de paz}

Tal carencia se debe en parte a la falta de claridad conceptual mencionada, pero también a factores institucionales y políticos, en concreto, a los diversos criterios existentes entre los órganos de la UE y a los diferentes intereses y agendas de sus Estados. En otras palabras, la prioridad dada a la misma varía sensiblemente entre unas instituciones comunitarias y otras, entre unos Estados y otros: hay quienes priorizan la cooperación al desarrollo, la seguridad humana o el refuerzo de la sociedad civil, y quienes prestan más importancia a la seguridad militar o los intereses económicos y comerciales. En suma, cabe afirmar que el modelo de construcción de paz de la UE está todavía en formación y cuenta con objetivos muy amplios y un tanto imprecisos (Castañeda, 2014:31).

\subsection{Persistencia de la fragmentación y de la falta de liderazgo claro}

El marco de construcción de la paz de la UE no es un modelo único, estático y coherente, sino fragmentado y mal coordinado, que refleja procesos de negociación complejos e incluso discrepancias sobre sus objetivos (Richmond et al, 2011:452). Estas deficiencias son características en gran medida del conjunto de la política exterior europea, que históricamente ha afrontado una falta de liderazgo claro. No en vano, la UE es una estructura compleja, compuesta por una diversidad de Estados y de órganos, que tienen diferentes discursos, agendas e intereses, lo cual dificulta consensuar y coordinar una política exterior común.

Como hemos visto, la UE ha realizado esfuerzos para afrontar este problema, con la formulación de los enfoques integral y, después, integrado, y la creación de sucesivos órganos para mejorar la coordinación. Aún así, sus diferentes políticas siguen siendo en buena medida compartimentos estancos a los que les falta una visión holística. Igualmente, existen dificultades de coordinación entre los actores y capacidades civiles y militares dentro de las 
misiones PCSD, hasta el punto de que es más habitual desarrollar sinergias con otros actores ajenos a la UE, como NNUU o la OTAN (Juncos y Blackmans, 2018:135-6).

Davis hace hincapié, sobre todo, en la falta de un liderazgo que le proporcione a la UE una guía estratégica para la prevención de conflictos. Según dice, existen fondos para la prevención a corto y largo plazo, así como mecanismos de coordinación entre el SEAE y la DEVCO. Sin embargo, añade, el principal reto es contar con el liderazgo político necesario para fijar prioridades e integrar la prevención de conflictos en la toma de decisiones estratégicas; en otras palabras, priorizar la prevención de conflictos a largo plazo por encima de la respuesta a crisis ya existentes (Davis, 2018:166-7).

\subsection{Falta de capacidades y recursos}

Uno de los problemas estructurales de la construcción de la paz de la UE sigue siendo la falta de capacidades y recursos, lo cual revela una insuficiente voluntad política de los Estados miembros para dar suficiente prioridad a este campo.

En el plano financiero, más que una falta de fondos potenciales existe una creciente presión para destinarlos a otros fines. Así, por ejemplo, el Instrumento en pro de la estabilidad y la paz (IcSP), dedicado a la respuesta a las crisis, ha sido utilizado para financiar el acuerdo entre la EU y Turquía diseñado para evitar la inmigración al continente (Davis, 2018:165).

Otro problema consiste en la limitación de personal y la de sus capacidades. Dado que el SEAE prioriza habitualmente la respuesta a crisis existentes, es insuficiente el número de expertos en prevención de conflictos. La presión para gestionar cuantiosos volúmenes de recursos impide que se le dedique el tiempo necesario a realizar análisis de los contextos de conflicto (Davies 2018:163). Además, la alta rotación del personal limita su conocimiento del contexto, sociedad y lenguas locales (Edmunds, Juncos y Algar-Faria, 2018:233), al tiempo que dificulta la acumulación de una memoria institucional de buenas prácticas en la materia (EPLO 2020:15).

\subsection{Escasa prioridad a la prevención y al afrontamiento de las causas raíces}

En el campo de los estudios de paz, la prevención de conflictos abarca diversas acciones orientadas a afrontar las causas tanto inmediatas como profundas de los conflictos para que no estallen en violencia. Un enfoque preventivo, por tanto, tiene un alcance más ambicioso que un enfoque reactivo, orientado a la mera gestión de crisis que ya han estallado. Al mismo tiempo, 
resulta económicamente más eficiente: se ha calculado que cada dólar invertido en prevención reduce en una media de 16 dólares los costes causados por el futuro conflicto (Luengo-Cabrera y Butler, 2017).

La UE ha conformado un discurso según el cual su estrategia de construcción de la paz se basa en un enfoque preventivo. Son numerosos los documentos que afirman tal perspectiva preventiva de la política exterior europea, como el Programa de Gotemburgo de 2001, el Tratado de Lisboa de 2009, las Conclusiones del Consejo sobre prevención de conflictos de 2011, o la Estrategia Global de 2016. Además, diferentes documentos señalan que la prevención requiere afrontar diversas "causas raíces" estructurales que pueden desencadenar violencia, como la pobreza, el abuso de derechos humanos y la exclusión de determinados grupos (Davis, 2018:158).

A pesar de este discurso, en la práctica la UE no parece realizar una apuesta clara por las acciones de prevención a largo plazo. En su lugar, prioriza las acciones de prevención temprana (early preventive action), de respuesta rápida y a corto plazo ante conflictos que ya han estallado (Davis, 2018:157). Según concluyen Pogodda et al, las intervenciones de la UE se basan en un modelo de "paz como gobernanza", es decir, promueven reformas de la gobernanza política y económica, pero no afrontan directamente el contexto político o las asimetrías de poder que son causas subyacentes del conflicto. Este enfoque, al mejorar la gobernanza y las condiciones socioeconómicas, contribuye al aplacamiento de la sociedad afectada y a la estabilización, pero no a resolver las causas raíces del conflicto (Pogodda et al, 2014:227).

Parece evidente que falta una apuesta política clara a favor de la prevención de conflictos. Cabe recordar que los procesos de toma de decisión en la UE son complejos y que la disparidad de visiones e intereses dificulta una estrategia común. Además, el Consejo apuesta por las acciones reactivas, siendo reticente a enfoques preventivos orientado a soluciones a largo plazo (Barrios, 2018:389, 395), dado que estas con frecuencia no reciben ni apoyo político ni recursos suficientes por parte de los Estados (Davis, 2018:394). A esto hay que añadir la persistente fragmentación institucional, pues diferentes órganos de la UE centrados en ámbitos que inciden en la prevención (como el comercio o la seguridad) siguen actuando en parte por separado y al margen de una perspectiva holística. Y, por último, la prevención a largo plazo se ve entorpecida por la falta de un liderazgo político que le proporcione la dirección y el peso necesarios. En efecto, la mayoría de los recursos en la materia están en las direcciones de la Comisión, pero sin embargo a esta no se le ha conferido el liderazgo en este campo, que descansa en el SEAE y, sobre todo, en el Consejo, por la reticencia de algunos Estados a que asuma un peso excesivo (Davis, 2018:161-2). 


\section{Conclusiones}

En las últimas décadas, la UE ha ido desarrollando un marco institucional para la prevención de conflictos y la construcción de la paz crecientemente sofisticado. No descansa en una institución específica, sino en un entramado de organismos, políticas e instrumentos financieros que operan en ese campo. Esta multiplicidad, precisamente, es una de sus principales fortalezas, pues le permite actuar en diferentes ámbitos y en todas las fases del conflicto.

Sin embargo, tal marco institucional, todavía en maduración, presenta también varias deficiencias, entre las que destacan su falta de claridad conceptual; la brecha entre un discurso normativo, transformador y preventivo de conflictos, y una práctica que prioriza la reacción a las crisis y la mera estabilización; y la fragmentación y descoordinación que persiste entre las diferentes instituciones y políticas en la materia.

En este sentido, cabe reconocer los importantes pasos que la UE ha dado en las últimas décadas para dotar de mayor coordinación a sus políticas en la materia. Entre ellos destaca la adopción de un enfoque integral y, más recientemente, un enfoque integrado para lograr que sus políticas se formulen y ejecuten de forma más coherente. Así como varias reformas institucionales, sobre todo la creación en 2019 de la Dirección ISP, que ha fusionado otros órganos y funciones.

A pesar de estos innegables avances, el camino hacia una mayor coherencia está todavía lastrado por varios obstáculos. Así, algunas políticas de la UE importantes para los intereses de sus Estados, como son la comercial y la crediticia del BEI, se resisten a ser integradas en un enfoque holístico y a asumir criterios de sensibilidad al conflicto. Algunos sectores son reticentes a utilizar la cooperación al desarrollo para afrontar las causas raíces de los conflictos. Y, sobre todo, el Consejo y los Estados siguen priorizando las actuaciones reactivas de gestión de crisis y se resisten a integrarlas con medidas y objetivos de prevención a largo plazo. Tal priorización cuestiona en parte el carácter de potencia normativa de la UE y revela una política exterior más centrada en la defensa de los intereses y la seguridad europea, orientación que ha cobrado fuerza con la nueva Estrategia de Seguridad de 2016.

Además, la creación de la Dirección ISP solo en parte ha contribuido a un enfoque más integrado. Ciertamente ha permitido mejorar la coordinación técnica y operativa dentro de la UE en la respuesta a los conflictos, por ejemplo mediante la fusión de las divisiones del SEAE con funciones civiles y militares en materia de seguridad, defensa y prevención de conflictos. Sin embargo, no ha logrado superar la brecha entre la dimensión operativa y la política. De hecho, apenas ha logrado involucrar en sus actividades y funciones a los Estados y a las direcciones geográficas de la UE. 
Probablemente la principal dificultad para hacer efectivo el enfoque integrado radica en que sigue faltando un liderazgo político común, que marque prioridades y articule con coherencia los diferentes instrumentos y políticas; que haga uso efectivo de la información y análisis generados por el sistema de alerta temprana; y, sobre todo, que aporte la visión y el impulso político necesarios para apostar por medidas preventivas a largo plazo.

En suma, a pesar de los avances logrados, la UE todavía no ha conseguido forjar un marco institucional cohesionado e integrado, que permita aprovechar plenamente el fuerte potencial que tiene para implementar políticas en diferentes ámbitos y en todas las fases del ciclo del conflicto, priorizando no la reacción ante su estallido, sino el afrontamiento de sus causas raíces con una perspectiva de largo plazo. 


\section{Referencias bibliográficas:}

Barbé, E. y P. Morillas (2019), “The EU Global Strategy: the Dynamics of a More Politicized and Politically Integrated Foreign Policy", Cambridge Review of International Affairs, 32 (6), pp. 753-770.

Barrios, L., "El uso de herramientas y mecanismos de construcción de paz por parte de la Unión Europea en países terceros: estado del arte y desafíos existentes", en GRASA, R. (dir.) (2018), Los retos de gestión de las violencias directas no políticas y la construcción de la paz, ICIP Reseach, $\mathrm{n}^{\mathrm{o}}$ 5, Barcelona, ICIP.

Bell, E. (2009), Society in State-building: Lessons in Improving Democratic Governance. Synthesis Report, Initiative for Peacebuilding, Bruselas.

Castañeda, D. (2014), The European Approach to Peacebuilding. Civilian Tools for Peace in Colombia and Beyond, Londres, Palgrave.

Churruca, C. (2017), "Desafíos y límites de la contribución de la Unión Europea a una sociedad internacional más pacífica y humana", en Alcaide, J. y Petit, E. (eds.), España y la Unión Europea en el orden internacional. XXVI Jornadas ordinarias de la AEPDIRI, Universidad de Sevilla, 15 y 16 de octubre de 2015, Tirant lo Blanch, Valencia, pp. 941-970.

Comisión Europea (2001), Comunicación de la Comisión Europea relativa a la prevención de conflictos, COM (2001) 211 final, Bruselas, 11 de abril.

Comisión Europea y Alta Representante de la Unión Europea para Asuntos Exteriores y Política de Seguridad (2013), El enfoque integral adoptado por la UE en relación con los conflictos y las crisis exteriores. Comunicación Conjunta al Parlamento Europeo y al Consejo, JOIN (2013) 30 final, Bruselas, 11 de diciembre.

Consejo de la Unión Europea (2003), Estrategia europea de seguridad: Una Europa segura en un mundo mejor, 15895/2003 PESC 787, Bruselas, 8 de diciembre.

Consejo Europeo (2001), Programa de la UE para la prevención de conflictos violentos, Consejo Europeo, Gotemburgo, 9537/1/01 REV 1 + REV 1 COR 1 (fi), junio.

Consejo Europeo (2007), Tratado de Lisboa por el que se modifican el Tratado de la Unión Europea y el Tratado Constitutivo de la Comunidad Europea, Lisboa, 13 de diciembre (2007/c 306/01).

Council of the European Union (2009), Concept on Strengthening EU Mediation and Dialogue Capacities, 15779/09, Brussels, 10 de Noviembre.

Davis, L. (2018), "Betwixt and between: conceptual and practical challenges of preventing violent conflict through EU external action", Global Affairs, 4 (2-3), pp. 157-169. 
Debuysere, L. y S. Blockmans (2019), Europe's Coherence Gap in External Crisis and Conflict Management. The EU's Integrated Approach between Political Rhetoric and Institutional Practice. Bertelsmann Stiftung, Gütersloh (Alemania).

Díez, T., S. Stetter y M. Albert (2006), "The European Union and Border Conflicts: the Transformative Power of Integration", International Organization, 60 (3), pp. 563-593.

Duke, S. (2011), The EU and Conflict Prevention: A Ten Year Assessment, Bonn, Working Group on Peace and Development (FriEnt).

EPLO (2014), Support for Peacebuilding and Conflict Prevention in the EU'S External Financing Instruments 2014-2020, EPLO Briefing Paper 1, Bruselas.

EPLO (2020), Mapping of Actors: The EU and Peacebuilding after Lisbon, Bruselas.

European Commission (2001), Communication from the Commission on Conflict Prevention, $\operatorname{COM}(2001)$ 211, 11 de abril.

Faleg, G. (2018), "The EU: from Comprehensive to Integrated Approach", Global Affairs, 4 (2-3), pp. 171-183.

Faria, F. (2014), What EU Comprehensive Approach? Challenges for the EU Action Plan and Beyond, ECDPM Briefing Note, $\mathrm{n}^{\circ}$ 71, Bruselas.

Hadden, T. (ed.) (2009), A Responsability to Assist. Human Rights Policy and Practice in European Crisis Management Operations, COST Report, Oxford.

Hauck, V. (2020), The Latest on the European Peace Facility and What's in it for the African Union, ECDPM Briefing Note, $\mathrm{n}^{\circ} 120$, Bruselas.

Hauck, V. y A. Sherriff (2013), Important Progress, but Real EU Comprehensiveness is Still Ahead of Us, ECDPM, Bruselas.

Juncos, A. E. y S. Blockmans (2018), "The EU's role in Conflict Prevention and Peacebuilding: Four Key Challenges", Global Affairs, 4 (2-3), pp. 131-140.

Luengo-Cabrera, J. y T. Butler (2017), "Reaping the Benefits of Cost-effective Peacebuilding', Global Observatory, 31, International Peace Observatory.

Manners, I. (2008), "The Normative Ethics of the European Union", International Affairs, 84 (1), pp. 45-60.

Martín de Almagro, M. (2017), Transitional Justice and Women, Peace and Security: A critical reading of the EU Framework, LSE Working Paper Series, $\mathrm{n}^{\mathrm{o}} 16$, Londres,

Tardy, T. (2017), The EU: from Comprehensive Vision to Integrated Action, EUISS, París. 

El inacabado camino de la fragmentación hacia la integración

Pogodda, S., O. Richmond, N. Tocci, R. Macginty y B. Vogel (2014), “Assessing the Impact of EU Governmentality in Post-conflict Countries: Pacification or Reconciliation?", European Security, 23 (3), pp. 227-249.

Richmond, O., A. Björkdahl y S. Kappler (2011) "The Emerging EU Peacebuilding Framework: Confirming or Transcending Liberal Peacebuilding?", Cambridge Review of International Affairs, 24 (3), pp. 449-469.

Unión Europea (2016), Una visión común, una actuación conjunta: una Europa más fuerte. Estrategia global para la política exterior y de seguridad de la Unión Europea, Servicio Europeo de Acción Exterior, Bruselas. 
